\title{
¿POR QUÉ SALIR A COMER? EN BUSCA \\ DE MODELOS QUE EXPLIQUEN LAS MOTIVACIONES DE COMER EN RESTAURANTES
}

\author{
EDUARDO JIRÓN FAJARDO \\ Escuela Ciencias de la Administración \\ Universidad Estatal a Distancia \\ ejiron@uned.ac.cr
}

\section{RESUMEN}

Existen diversos modelos que analizan y explican las variables que intervienen en la decisión de una persona para salir a comer fuera. Se realizó una investigación exploratoria, en una zona de San José, por medio de la aplicación de una encuesta basada en dos modelos para determinar, desde el propio consumidor, su perspectiva de calidad, servicio y satisfacción en su experiencia de comer fuera. Se realizó un análisis correlacional de esas experiencias o expectativas previas, de recibir deleite con una respuesta de algo deseable a repetirse, bajo el supuesto que sea la variable principal en su decisión de volver a un restaurante. Se logra conocer las razones o motivaciones que una persona tiene para salir a comer en restaurantes de servicio completo.

PALABRAS CLAVE: EXPERIENCIA DEL CONSUMIDOR, COMIDA, EMOCIONES DE CONSUMO, RESTAURANTES DE SERVICIO COMPLETO.

\section{ABSTRACT}

There are several models that analyze and try to explain the variables involved in the decision of a person for eating out. Exploratory research was conducted in an area of San Jose, through the implementation of a survey based on two models to determine, from the consumer, their perspective of quality, service, and satisfaction in their experience of eating out. A correlational analysis was conducted of those experiences or prior expectations, delight in receiving a response from desirable to repeat, assuming that is the main variable in their decision to return to a restaurant. It is possible to know the reasons that motivate a person to go eating out at full service restaurants.

KEYWORDS: CONSUMER EXPERIENCE, FOOD, EATING OUT, CONSUMER EMOTIONS, FULL-SERVICE RESTAURANTS.

\section{INTRODUCCIÓN}

La comida está relacionada con valores culturales, religiosos o sociales en distintos pueblos y naciones; se le ha dado un lugar preponderante para acompañar eventos de importancia, según el caso (bodas, bautizos, negocios, cumpleaños, graduaciones, entre otros). Después de revisar estudios relacionados con el tema de salir a comer (eating out, dining out), se encuentran numerosas investigaciones al respecto enfocadas desde múltiples puntos de vista, así como las mismas variables que inciden en esta actividad. Por tanto, se decide realizar la aplicación de un modelo para indagar sobre las motivaciones de las personas que salen a comer en restaurantes de servicio completo.

Schembri y Sandberg (2011) se refieren al concepto de "fenomenografía" para afirmar que los clientes (o consumidores) evalúan la calidad del servicio desde el propio significado de calidad de servicio que dicho consumidor posee y además de la experiencia de la calidad de servicio en un contexto de servicio particular. 
Lo que todos estos modelos van encontrando en común es que la calidad del servicio es un conjunto de dimensiones fijas que se originan de las expectativas y percepción del consumidor. Alonso y O’Neill (2010) exponen que la decisión de visitar uno u otro restaurante puede determinarse por distintos comportamientos del consumidor con respecto a su reacción ante los distintos componentes y patrones de restaurantes que pueden ser, hasta cierto punto, controlados por la gerencia. También se menciona que los distintos grupos de edades de manera similar tienen su impacto en la selección del tipo de restaurante donde ir a comer. Se rescata el enfoque dado al ambiente para influenciar la percepción del cliente sobre la calidad del servicio, ya que genera una impresión y respuesta emocional de parte del cliente. Por otro lado, Acharya, Patterson, Schmitz, Foerster, Hill, Jones y Bohm (2008) toman en consideración la actitud positiva de las personas hacia comidas sanas, como consumidores predispuestos para seleccionar un menú sano y, por ende, adquirir (o comprar) un producto de dicho menú.

En otro estudio, realizado por Howlett, Burton, Bates y Huggins (2009) en Estados Unidos, se analiza el comportamiento de consumo y la evaluación al menú del consumidor influenciada por la información sobre nutrición que presentan los menús en los restaurantes que se realizó el experimento.

No obstante, con el afán de encontrar un modelo que considere de manera integral diversos criterios, se continuó buscando literatura adicional que pudiese servir de asidero para responder a la pregunta precitada, se encuentran los enfoques de otros estudiosos adicionales. Anderson y Mossberg (2004) rescatan las bases de las emociones para tratar de responder a su pregunta inicial de porqué la gente consume, y exploran en su estudio el grado en que los restaurantes satisfacen a sus clientes.
La investigación de Anderson y Mossberg (2004) se basa en lo que sucede en el sistema de activación reticular ascendente del cerebro medio, el cual atraviesa los tonos hedónicos (positivo y negativo) que buscan la comodidad. Llevan dicho trabajo hasta caer en un modelo que considera tres necesidades generales (fisiológica, social e intelectual), las cuales pueden ser satisfechas en cierto grado por productos y servicios que la persona consume o experimenta.

El modelo cita que los restaurantes pueden satisfacer las necesidades mencionadas en la medida que se alcance un buen grado de desempeño de los factores que Anderson y Mossberg (2004) llaman "factores influenciantes de la experiencia de los comensales" donde pone como base un buen producto (la comida que "debe ser" de buena calidad) y cinco factores adyacentes (servicio, buena cocina, interior del restaurante, buena compañía y otros clientes), en dependencia del alcance de estos factores, plantean que son satisfactores o deleitadores. Concluyen que la variedad de criterios hace posible el grado de éxito de un restaurante en proveer satisfacción a la necesidad de sus consumidores.

Por lo tanto, ante la respuesta del modelo anterior que se limita al hecho de lograr con éxito la satisfacción de necesidades en el cliente, es que se opta por otro modelo. El de la comida de los cinco aspectos (ver figura 1) que según Edwards y Gustafsson (2008), ha sido utilizado con éxito durante años para enseñar el arte y la ciencia de los servicios de comida. Igualmente, en dicho estudio se hace mención a que no solo es el platillo el que influencia el disfrute de una ocasión de comida, sino que hay factores contextuales que se dividen en dos: simultáneos y temporales. 


\section{FIGURA 1}

\section{MODELO CINCO ASPECTOS DE LA COMIDA (FAMM*)}

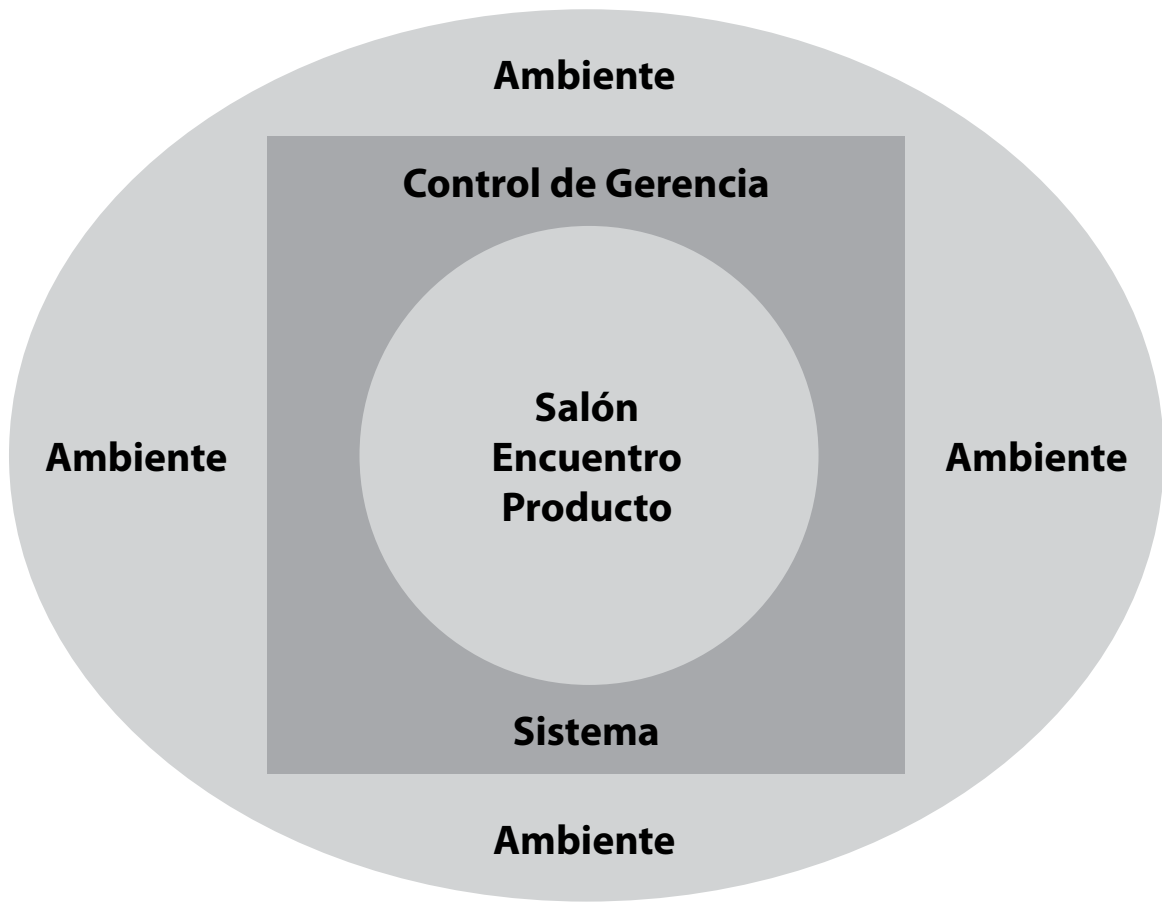

Fuente: Edwards y Gustafsson (2008).

* Sus siglas en inglés: Five Aspect Meal Model.

En este modelo se materializan o traducen esos factores a elementos que conforman parte de la actividad diaria en un restaurante. Cada uno de estos temas es definido y servirá de base para determinar las variables del estudio. Su relevancia está en la experiencia de los clientes al visitar un restaurante para disfrutar, además del platillo, todo el ambiente ahí circunscrito.

Se opta por aplicar dicho modelo porque ahí se materializa de manera muy concreta, tanto los elementos a considerar en un restaurante como, también los factores que explican Anderson y Mossberg (2004) en su modelo como respuesta a las tres necesidades genéricas que motivan a todo ser humano y que ambos modelos concuerdan en que una comida en un restaurante (eating out, dining out) se convierta en una experiencia disfrutable, satisfactoria hasta el nivel de deleite, lo cual la hace por sí misma una motivación para querer repetirla.

Una persona que está motivada por algún agente (elemento o necesidad) a salir a comer, va a un determinado lugar con cierta expectativa y si realmente logra satisfacer ese agente motivador, su expectativa fue superada y repite la experiencia. La intención es recoger toda esa información con algún modelo donde luego podamos analizar sus variables e interrelaciones.

Realizar el presente estudio será un aporte de conocimiento que no está siendo cubierto en este momento por ninguno de los exponentes en el sector de restaurantes, lo cual implica información relevante que podrá servir de base para eventuales estrategias de mercadeo o quizá para establecer algún eventual nuevo concepto para satisfacer ese porqué de salir a comer. 
Adicionalmente, se debe tomar en cuenta que el nivel de gastos de la población, residente en Costa Rica, consumiendo bebidas y comidas fuera del hogar ha crecido en un 58,94\% de 1998 a 2004, según lo consultado en el boletín de la Encuesta Nacional al Hogar del INEC (ver Tabla 1). Esta tendencia deja claro las oportunidades para los empresarios del sector gastronómico de atraer comensales que visitan el tipo de establecimiento explorado en esta investigación.

Realizando algunas estimaciones (ver Cuadro 76 de ENAHO-INEC, Ingresos y Gastos 2006, para datos completos) se puede comparar el consumo de bebidas y comidas fuera del hogar tanto en la zona urbana como en la zona rural para el año 2004, observando en 7,3\% y 8,0\% el porcentaje respectivo de consumo; siendo levemente mayor en la rural.
Por tanto, se vuelve interesante para las ciencias del Mercadeo, por medio del análisis en el comportamiento de las variables del modelo, llegar a conocer a mayor profundidad las motivaciones de los comensales y así poder brindar información de provecho para una mejor toma de decisiones estratégicas a los empresarios de ese nicho de restaurantes.

Especialmente, por el incremento en la actividad económica de dicho sector, según cita el reporte del Banco Central de Costa Rica (2011):

...Las industrias que integran el grupo comercio, restaurantes y hoteles alcanzaron un crecimiento de 4,7\%, continuando así con el dinamismo observado en el 2010. Este comportamiento es el resultado de estrategias de mercadeo impulsadas principalmente por el comercio, dirigidas a los es-

TABLA 1

\section{GASTO DE CONSUMO PER CÁPITA SEGÚN GRUPO DE GASTO(EN COLONES) 1998-2004}

\begin{tabular}{lcccc}
\multicolumn{1}{c}{ Zona y grupo de gasto } & Año1988 & Año 2004 & Variación porcentual & Proporción del gasto \\
TOTAL PAís & & & & \\
Personas & 2832543 & 4267262 & 50,65 & \\
Gasto de consumo & 47313 & $\mathbf{6 8 3 1 4}$ & $\mathbf{4 3 , 3 9}$ & $22,3 \%$ \\
Alimentos, tabaco y bebidas & 15741 & 15232 & $-3,23$ & $6,9 \%$ \\
Prendas de vestiry yalzado & 4458 & 4719 & 5,86 & $14,5 \%$ \\
Alquiler de vivienda, agua y electricidad & 5742 & 9906 & 72,52 & $8,9 \%$ \\
Muebles, artículos para el hogar y otros & 5153 & 6093 & 18,24 & $4,8 \%$ \\
Salud & 1736 & 3256 & 87,56 & $18,3 \%$ \\
Transporte y comunicaciones & 5478 & 12483 & 127,87 & $10,1 \%$ \\
Educación, cultura y esparcimiento & 2082 & 6873 & 230,11 & $7,5 \%$ \\
Comidas y bebidas fuera del hogar & 3227 & 5129 & 58,94 & $6,8 \%$ \\
Bienes y servicios diversos & 3694 & 4622 & 25,12 &
\end{tabular}


tratos medios, destacando las facilidades de financiamiento para la compra de vehículos y electrodomésticos. Las actividades de hoteles y restaurantes se han beneficiado del aumento en el turismo nacional y extranjero, debido a la recuperación de la actividad económica así como de la adopción de políticas promocionales (p. 4).

\section{MARCO TEÓRICO}

Varios de los modelos que se encontró en la literatura, entre ellos los empleados en el presente estudio, se basan en las teorías sobre las que se basa el conocido triángulo de Maslow. Entre las necesidades básicas, que acuerpa dicho modelo, están las fisiológicas; también otras como las intelectuales y sociales. Sin embargo, se encontró que ha habido una evolución, a través de las investigaciones relacionadas, donde los autores fueron encontrando importantes elementos que impactan en la experiencia de comer fuera y por tanto, los estudios han adicionado paulatinamente más factores.

Recorriendo hacia atrás los hallazgos, previo a los estudios Gustafsson en 2004, la literatura referencia los de Rozin y Tuorila (1993), donde exponen variables contextuales que influencian el nivel de satisfacción que experimenta una persona que sale a comer, quienes los categorizan en dos tipos de factores: 1) contextual simultáneo, por ejemplo, el sabor de un simple alimento cuando es comido con otros; 2) contextual temporal, sean acciones o factores pasados o futuros, los cuáles pueden cruzar por la mente de una persona durante el evento de comer, por ejemplo, la aceptación de un simple alimento, el cuál declina a medida que es consumido.

Según Gustafsson (2008), anteriormente otros estudiosos (Meiselman et al. 1988; Meiselman, 1996) encuentran una clasificación alternativa sobre las influencias alrededor del lugar don- de son consumidos los alimentos y les llaman variables situacionales. Estas son consideradas importantes para el consumo y aceptación de una comida. Al mismo tiempo, había científicos sociales como Wood (1990), citado por el mismo autor, que usualmente clasificaban estas circunstancias como factores potenciadores o restrictivos, lo cuales incluyen aspectos como la disponibilidad económica para comer fuera, las habilidades sociales apropiadas.

Según Belk (1974, citado por Gustafsson, 2008) preguntas como "¿Dónde prefiere comer?" y sus respuestas bien podrían ser "en dependencia de las situaciones" quién resume muy bien sobre las variables situacionales con el texto siguiente:

... investigaciones del comportamiento del consumidor, quién ignora efectos situacionales son como resultado en buenas predicciones solamente cuando las características de las alternativas de escogencia del comprador son suficiente intensas para ser influenciadas a través de todas las situaciones relevantes (p. 4).

Existe referencia de otros estudios a inicios de 1945, sobre otros factores que influencian el comer fuera, por tanto, hay noción que el tema no es nuevo; cuando la aceptación de mediciones de alimentos, tanto individual como por clases, fueron demostradas ser diferentes al consumirse en la tierra o en un avión. La comida era más crítica en los vuelos, donde las menores alteraciones de apariencia, color y sabor tuvieron una considerable afectación en aceptabilidad (Green y Butts, 1945).

También se utiliza en el presente análisis, el trabajo de Ryu (2005) respecto a las variables moderadoras, las cuáles influyen en la variable dependiente, lo cual se describe en la pregunta de si hay otras motivaciones que impactan en dicha experiencia del comensal para hacerla gratificante o no. Así que también se evaluaron en el presente estudio, y con base en la literatu- 
ra revisada, se indica que en efecto hay diversas motivaciones que una persona tiene para salir a comer.

El estado de ánimo del cliente tiene su efecto en la percepción de esa experiencia; dicho estado puede influirse por estimulación durante el tiempo de permanencia en el local. El modelo que servirá para evaluar esto es el de MehrabianRussell, que data de 1974 según Ryu (2005), y se esquematiza en la figura 2: se utiliza el paradigma Estímulo-Organismo-Respuesta (S-O-R*).

Figura 2

MODELO DE MEHRABIAN-RUSSELL

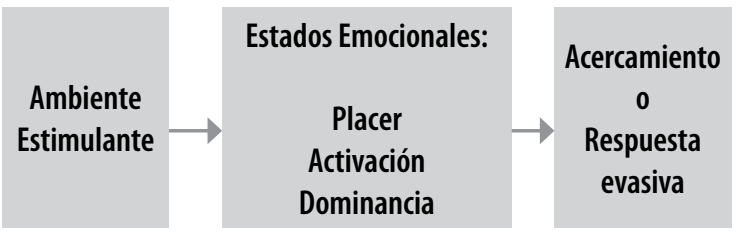

Fuente: Ryu (2005)

Las tres respuestas emocionales son placer, activación y dominancia; donde placer (contentomolesto) es un estado de afecto positivo que se evidencia en la preferencia, gustar, refuerzo positivo. Arousal (activado-inactivado) se refiere al estado ánimo que varía a lo largo de la dimensión desde somnoliento hasta excitación frenética. Por último, dominancia (dominantesumiso) se basa en la medida de sentirse libre para actuar en diversas formas.

Según Ryu (2005), el modelo postula que todo ambiente generará un estado emocional sobre el individuo en término de los tres estados emocionales, los cuáles son factorialmente ortogonales. Dado esto último y considerando lo que expone Tabachnik (capítulo 1) podremos emplear los análisis multivariados para determinar

* Siglas del inglés: Stimulus-Organism-Response. la influencia de los predictores sobre la variable dependiente que nos interesa conocer la "Experiencia de salir a comer" explicada gráficamente en la figura 3.

\section{FIGURA 3}

\section{EFECTO DE LOS PREDICTORES SOBRE LA“EXPERIENCIA DE SALIR A COMER}

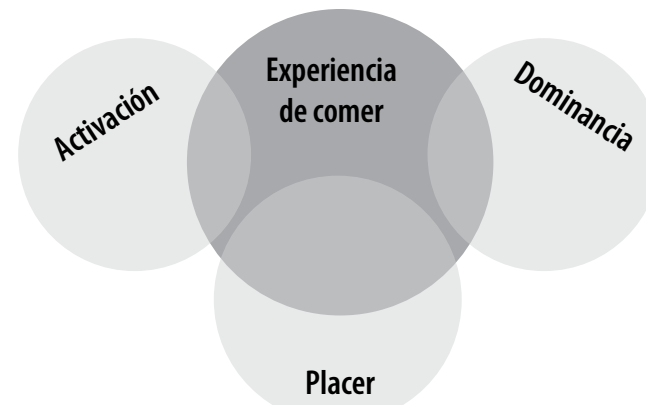

Fuevte: elaboración propia, basado en modelo de Mehrabian-Russell.

Han, Back y Barrett (2008) explican en su estudio que el modelo de Mehrabian-Russell desarrolló la escala placer-arousal-dominancia (PAD), con lo cual se pretende poder relacionar cuánto impacta en su respuesta (Estado emocional), la satisfacción de las tres necesidades.

Las tres necesidades que comentan Anderson y Mossber (2004) son: necesidades fisiológicas, sociales e intelectuales que serán nuestras variables moderadoras.

El grupo de variables (ver Tabla 2) del PAD son a la vez dependientes (de la satisfacción-necesidades) y moderadoras (sobre el consumo). Este modelo fue desarrollado sobre experimentación en restaurantes de alto nivel.

Aunque los tipos de emociones son mucho más, se toman solamente las situaciones que se relacionan con experiencias de clientes en un restaurante; entonces, al final la experiencia que perciba el comensal va a tener su impacto en el consumo. Al hablar de consumo, requeriremos medirlo por medio de sus variables tales como: intensidad, frecuencia, intencionalidad, variedad y ocasión. 
TABLA 2

VARIABLES DEL PAD

\begin{tabular}{|c|c|c|c|}
\hline EMOCIÓN & COMODIDAD & MOLESTIA & ROMANCE \\
\hline Excitado & Cómodo & Irritado & Romántico \\
\hline Sorprendido & Contento & Frustrado & El amor \\
\hline Asombrado & Amistoso & Decepcionado & Sentimental \\
\hline Curioso & Relajado & Enojado & \\
\hline Sofisticado & Satisfecho & Escéptico & \\
\hline Interesado & Respetado & & \\
\hline Esperanzado & Feliz & & \\
\hline Emocionado & Cumplido & & \\
\hline Agradecido & Cálido & & \\
\hline Apasionado & Seguro & & \\
\hline Entretenido & & & \\
\hline Entusiasta & & & \\
\hline Activado & & & \\
\hline Mimado & & & \\
\hline
\end{tabular}

Fuevte: traducción por el autor, de Han et al. (2008).

El trabajo exploratorio aquí desarrollado, acerca de modelos utilizados en otros países, describe la aplicación de estos en Costa Rica y conduce a buscar una explicación científica en la percepción y motivación de personas que comen fuera de casa. El sector gastronómico de restaurantes movió en el año 2004, un volumen de colones de 21.888.916.453 (Tabla 1, multiplicando el rubro "Comidas y bebidas fuera del hogar" por el de "personas") nada despreciable, el cuál es un 7.5\% del total gastos de consumo de dicho período.

Tanto de la teoría como de la práctica se conoce que el lugar donde un producto o servicio es ofrecido o consumido, tiene gran impacto en la decisión de compra. El sitio y más específicamente el ambiente (o la atmósfera) de este, puede influenciar más que el producto mismo (por ejemplo, una comida) en la persona que adquirirá el producto. Kotler indica (según cita de Ryu, 2005) que hay situaciones donde la influencia primaria puede ser el ambiente (atmósfera).

"La atmósfera es el esfuerzo para diseñar ambientes de compra que produzcan efectos emocionales en el consumidor que aumente su probabilidad de compra" (Kotler, 1973, p. 50, citado en Ryu, 2005). Retomando el modelo donde Kotler (véase Fig. 4) expone que hay influencia del ambiente en un restaurante para la decisión de compra del visitante.

Esa atmósfera, descrita en la figura 4 como "calidades sensoriales del espacio circundante"; es posible generarla a través de los sentidos. Los principales canales sensoriales, según Kotler (citado en Ryu, 2005), por medio de los cuáles se concreta la percepción del ambiente son: vista, sonido, esencia y tacto.

\section{LA CADENA CAUSAL CONECTANDO ATMÓSFERA Y PROBABILIDAD DE COMPRA}

\begin{tabular}{|c|c|c|c|}
\hline $\begin{array}{c}\text { Calidades } \\
\text { sensoriales del } \\
\text { espacio circundante } \\
\text { al objeto de compra }\end{array}$ & $\begin{array}{c}\text { Percepción } \\
\text { del comprador } \\
\text { de las calidades } \\
\text { sensoriales } \\
\text { del espacio }\end{array}$ & $\begin{array}{l}\text { Efecto de las } \\
\text { calidades sensoriales } \\
\text { percibidas sobre } \\
\text { el estado afectivo } \\
\text { e información } \\
\text { del comprador }\end{array}$ & $\begin{array}{l}\text { Impacto del estado } \\
\text { afectivo e información } \\
\text { modificada del } \\
\text { comprador sobre } \\
\text { la probabilidad } \\
\text { de compra }\end{array}$ \\
\hline
\end{tabular}

Fuente: Ryu (2005). 
En el presente trabajo, empleando un análisis correlacional, se encuentran las principales variables de influencia en la decisión de comer fuera para una muestra de población del área metropolitana, compuesta principalmente por cuatro provincias: San José $(69,5 \%)$, Heredia (15,2\%), Cartago (8,6\%) y Alajuela $(5,7 \%)$.

\section{METODOLOGÍA Diseño}

Es un estudio exploratorio mediante una investigación aplicada con enfoque cuantitativo, cuyo diseño y análisis son del tipo no-experimental y transversal respectivamente. La investigación fue realizada por medio de aplicación de encuestas en tres canales diferentes: algunos restaurantes del tipo de servicio completo del área de Montes-Oca, en lugares de trabajo de personas que laboran en San José y remitiendo el instrumento por medio de correo electrónico.

\section{Participantes}

La población consistió en personas adultas (20 años o más) con nivel de educación superior que residieran en el área metropolitana y se en- contraran en un restaurante, o bien personas que habían visitado un restaurante tipo serviciocompleto en el periodo de un año o menos al momento de aplicar el instrumento (finales del año 2011). El objetivo era contar con sujetos que tuviesen poder de decisión para salir a comer, además de considerar que fueran personas con algún nivel de ingresos generados por cuenta propia sin restringir la fuente (rentista, asalariado, empresario, pensionado u otro) y de preferencia profesional (actual o en mediano plazo).

La meta era obtener una muestra de mínimo 100 sujetos para poder realizar distintos análisis estadísticos utilizando SPSS y determinar las principales variables que intervienen en las decisiones de salir a comer a un restaurante del tipo precitado.

Se procesó un total de 105 instrumentos, siendo 74 mujeres y 31 hombres. El rango de edad entre los 20 y 66 años $(M=38,2$ y $D E=9,9)$; el grupo de casados es mayoría (50,5\%), seguido por solteros (38\%) y luego unión libre (7,6\%). El rango entre de hijos es entre 1 y $5(\mathrm{M}=1$ y $\mathrm{DE}=$ 1,16); mientras que el nivel de estudios del encuestado es universitario (39) correspondiente a $37,1 \%$; posgrado (38) con un valor de $36,2 \%$ y universitario inconcluso (21) equivalente a $20 \%$.

TABLA 3

\section{DESGLOSE DE ÍTEMS POR SECCIÓN}

\begin{tabular}{|l|c|l|}
\hline \multicolumn{1}{|c|}{ SECCIÓN } & ÍTEM & \multicolumn{1}{|c|}{ VARIABLES EVALUADAS } \\
\hline Variables sociodemográficas & 7 & Control para grupos diferentes \\
\hline Preferencias de esparcimiento o recreación & 9 & Necesidades a satisfacer relacionadas con el modelo FAMM \\
\hline Aspectos de un restaurante que son importantes para usted & 14 & Relacionar variables del modelo FAMM como estímulo para emociones \\
\hline $\begin{array}{l}\text { Reacciones diversas en función de la experiencia percibida en un } \\
\text { restaurante }\end{array}$ & 17 & Modelo PAD, relación de emociones con Experiencia de comer fuera \\
\hline Criterios relacionados con su vista a restaurantes de "servicio completo" & 12 & Distintas variables para poder medir el consumo \\
\hline Generalidades acerca de su tiempo y esfuerzo por visitar el restaurante & 11 & $\begin{array}{l}\text { Relacionar expectativas del cliente con una experiencia previa } 0 \text { al } \\
\text { momento y su percepción de salir a comer }\end{array}$ \\
\hline
\end{tabular}

Fuente: elaboración propia. 
Con respecto a los puestos de trabajo, $84,7 \%$ se acumula con 4 categorías de puesto encontrados: otro (40\%), coordinación (19\%), encargado $(18,1 \%)$ y dueño (7,6\%). En la categoría de "otro" se encontró a personas en puestos como asistente, empleado y secretaria, por ejemplo.

\section{Instrumentos}

Se utilizó el mismo instrumento, tanto en modalidad impresa como digital, ya que se aplicaban de manera presencial y por medio de correo electrónico. Dicho instrumento se compone de un total de 70 ítems agrupados en 6 secciones (ver desglose en Tabla 3). Las variables asociadas corresponden a modelos investigados (PAD, FAMM) durante la etapa de revisión de literatura.

El instrumento se diseña para medir 41 de las variables mediante escala (1 a 7), 20 combinando esa escala y selección única, y las 9 restantes con selección única. Estas últimas son principalmente variables con información demográfica de los encuestados.

Se analizan las variables de los modelos previamente explicados (FAMM y PAD), donde las categorías son: independientes, dependientes, moderadoras y mixtas por ser a su vez dependientes y moderadoras (ej. las del PAD: activación, dominancia y placer).

\section{Procedimiento}

Para obtener la información se aplicó entrevista, principalmente de manera directa a un $85 \%$ de la población y el resto se le envió la encuesta por correo electrónico. El tiempo de duración para responder cada cuestionario fue de aproximadamente 30 minutos, que en futuras experiencias es preferible reducir hacia las variables críticas encontradas de cara a la decisión del comensal.

Se pidió a los participantes completar la encuesta, tanto directamente a comensales en el mismo sitio visitado, como a otras personas en otro ambiente, como en su lugar de trabajo o en línea, y así traer a su memoria sus últimas salidas a comer y contar con el criterio de experiencias diferentes y no solo la recogida en los propios restaurantes visitados. En total, se aplicó a un total de 105 personas, cuya distribución puede verse en la Tabla 4.

TABLA 4

\section{DISTRIBUCIÓN DE INSTRUMENTOS POR GRUPO AL APLICAR ENCUESTAS}

\begin{tabular}{|l|l|l|}
\multicolumn{1}{|c|}{ SUJETOS } & \multicolumn{1}{|c|}{$\begin{array}{c}\text { INSTRUMENTO } \\
\text { UTILIZADO }\end{array}$} & \multicolumn{1}{c|}{ POBLACIÓN } \\
\hline Comensales & $\begin{array}{l}\text { Encuesta con } \\
\text { entrevista personal }\end{array}$ & 27 personas \\
\hline Personas no en restaurantes & $\begin{array}{l}\text { Encuesta con } \\
\text { entrevista personal }\end{array}$ & 63 personas \\
\hline Personas varias & Encuesta en línea & 15 personas \\
\hline
\end{tabular}

Fuente: elaboración propia.

Se tabularon algunos datos en preguntas abiertas ("permanencia-horas en un restaurante", "cómo hacen que el cliente se sienta con los aspectos que la gerencia controla" $y$ "la principal razón para salir a comer") donde había una amplia variedad de respuestas para obtener información más valiosa, ya que se logra clasificar por similitud.

Se contó con un conjunto de descriptores de emoción sobre el consumo y la repetición de estos es un indicador de qué tanto afectan al momento de consumir; utilizándose aquí "análisis multivariado" Análisis ANNOVA y ANCOVA.

Las variables-PAD (ver tabla2) se midió mediante escalas para conocer cómo actúan sobre los criterios de las variables relacionadas con el consumo. Con los datos obtenidos se construyó tablas de frecuencias para las variables como: género, estado civil, nivel-escolaridad, puesto-trabajo y 
ubicación geográfica. El grueso de las variables con escala fueron utilizadas para realizar análisis multi-variado (análisis descriptivos, correlación simple y múltiple, modelos de regresión simple y múltiple, anova y manova), análisis de factores y análisis de confiabilidad, todo lo anterior, apoyándose en el uso de paquetes estadísticos (software estadístico SPSS).

\section{RESULTADOS \\ Validación del modelo FAMM y del modelo PAD}

Se procedió de dos maneras para validar estos dos modelos: a) en un caso la base fue la teoría y por tanto, se tomaron las preguntas (con escala) asociadas con cada factor y se construyó uno a uno, utilizando una fórmula en el SPSS. De esta manera se calcularon los indicadores para este y otro modelo. b) en el otro caso, se utilizó análisis factorial para obtener los factores FAMM y PAD generados por la misma base de datos, donde se agruparon todas las mismas preguntas que se utilizaron en la parte anterior y se utilizó 0,6 como valor absoluto, pues se quiso dejar solamente las variables con fuerte correlación.

Se realizó la estimación de los estadísticos de todas las variables escala, excepto 5 que tenían opción abierta, del instrumento (Tabla 5) $y$, posteriormente, se ejecutó la confiabilidad para las preguntas respecto a las escalas, este resultado fue estimado de dos maneras: a) corrida general de todas las preguntas (total 56) y b) en corridas separadas, las preguntas con selección única y escala (20) y las preguntas con solo escala (36); en todos los casos el Alfa de Cronbach obtenido fue alto.
TABLA 5

\section{INSTRUMENTO: VARIANZA TOTAL EXPLICADA}

\begin{tabular}{|c|c|c|}
\hline $\begin{array}{c}\text { ALFA DE } \\
\text { CRONBACH }\end{array}$ & $\begin{array}{c}\text { ALFA DE CRONBACH BASADA } \\
\text { EN LOS ELEMENTOS TIPIFICADOS }\end{array}$ & $\begin{array}{c}\text { N. }{ }^{\circ} \\
\text { ELEMENTOS }\end{array}$ \\
\hline 0,89 & 0,91 & 56 \\
\hline 0,82 & 0,85 & 20 \\
\hline 0,86 & 0,88 & 36 \\
\hline
\end{tabular}

Fuente: elaboración propia.

En referencia al FAMM, en Tabla 6 se puede ver como 7 factores explican 61,8\% de la varianza en las 23 variables originales. Dichas variables corresponden a las preguntas indicadas en Tabla 7.

TABLA 6

\section{VARIANZA TOTAL EXPLICADA DEL MODELO FAMM}

\begin{tabular}{|c|c|c|c|}
\hline FACTOR & TOTAL & \% VARIANZA & \% ACUMULADO \\
\hline 1 & 3,52 & 14,1 & 14,1 \\
\hline 2 & 2,84 & 11,4 & 25,5 \\
\hline 3 & 2,46 & 9,84 & 35,3 \\
\hline 4 & 2,02 & 8,09 & 43,4 \\
\hline 5 & 1,69 & 6,79 & 50,2 \\
\hline 6 & 1,49 & 5,97 & 56,2 \\
\hline 7 & 1,41 & 5,66 & 61,8 \\
\hline
\end{tabular}

Fuevte: elaboración propia a partir del SPSS “Método de extracción: Análisis de Componentes principales

Luego del análisis, se encontró grupos de preguntas con fuerte correlación que correspondían a variables del modelo. Considerando esto, se eligió el nombre indicado para el factor correlacionado (véase Tabla 7).

Se tomó solamente cuatro de los factores, por cuanto, hay otros tres que tienen correlación fuerte solamente con una variable cada uno, lo cual no permitía agrupar preguntas. Por otra parte, hubo 10 preguntas que no mostraron una fuerte correlación con ninguno de los factores. 
TABLA 7

\section{CONFORMACIÓN DE FACTORES}

\begin{tabular}{|l|l|}
\hline \multicolumn{1}{|c|}{ Factores } & \multicolumn{1}{|c|}{ Preguntas } \\
\hline Ctrl. Gerencial & $\begin{array}{l}\text { Controles del restaurante } \\
\text { Instalaciones interiores } \\
\text { Forma de pago } \\
\text { Eficiencia como criterio de selección }\end{array}$ \\
\hline Contexto & $\begin{array}{l}\text { Presentación del producto } \\
\text { Ambiente ameno } \\
\text { Identificación con otros comensales }\end{array}$ \\
\hline Servicio integral & $\begin{array}{l}\text { Calidad del servicio } \\
\text { Confort y seguridad } \\
\text { Operación del salón }\end{array}$ \\
\hline Producto & $\begin{array}{l}\text { Producto en su estándar } \\
\text { Comida con bebida }\end{array}$ \\
\hline
\end{tabular}

Fuente: elaboración propia

Las preguntas que aparecen solas con interrelación fuerte con algún factor son: "Gusta de restaurantes por tener tecnología", "Visita mismo restaurante y pide mismo platillo"y "La interrelación con el personal hacen sentir bien".

Luego de realizar el análisis factorial del FAMM (control gerencial, contexto, servicio integral, producto); hubo 10 preguntas que no mostraron una fuerte correlación $r(105)=, 6 p<, 01$ con ninguno de los factores.

También se validó el modelo PAD (satisfacción de necesidades básicas), para lo cual se utilizaron las mismas condiciones que para el modelo anterior, ver los resultados en Tabla 8. Podemos notar que 6 factores explican 68,9\% de las 17 preguntas originales.

Dichas variables corresponden a las preguntas indicadas en el cuadro siguiente, en el que se eligió el nombre indicado para el factor correlacionado.
Se tomaron solamente tres de los factores, por cuanto, hay otros 3 que se correlacionan mucho pero solamente con una pregunta cada uno; por ejemplo, la pregunta "Mi placer por comer es alto"y lo curioso es que esta pregunta se relaciona con el factor generado "Comer"; no obstante, dicho factor no la explica.

TABLA 8

\section{VARIANZA TOTAL EXPLICADA DEL MODELO PAD}

\begin{tabular}{|c|c|c|c|}
\hline FACTOR & TOTAL & \% VARIANZA & \% ACUMULADO \\
\hline 1 & 3,59 & 21,2 & 21,2 \\
\hline 2 & 2,61 & 15,4 & 36,5 \\
\hline 3 & 1,86 & 10,9 & 47,5 \\
\hline 4 & 1,30 & 7,66 & 55,1 \\
\hline 5 & 1,17 & 6,90 & 62,0 \\
\hline 6 & 1,17 & 6,86 & 68,9 \\
\hline
\end{tabular}

Fuente: elaboración propia a partir del SPSS “Método de extracción: Análisis de Componentes principales

Al generarse el análisis factorial para el PADD (socializar, comer, recrearse), quedaron 2 preguntas sin ser explicadas por alguno de los 6 factores. Igualmente, llamó la atención el hecho que la pregunta "¿Tengo pasatiempo favorito para recrearme?" no tiene relación con el resto de preguntas que están asociadas con alimentos.

Posteriormente, con las preguntas que no estaban directamente asociadas con el modelo FAMM y PAD, se realizó otro análisis factorial, resultando que 5 factores explican 66.9\% de las 15 variables originales. Las respectivas preguntas igualmente a los modelos previos, también se agruparon por similitud que puede apreciarse en Tabla 9; nuevamente quedan preguntas sin ser explicadas por ningún factor, al nivel establecido de significancia $(0,6)$ 
TABLA 9

\section{FACTORES ASOCIADOS AL CONSUMO (ANÁLISIS FACTORIAL)}

\begin{tabular}{|l|l|}
\hline \multicolumn{1}{|c|}{ FACTORES } & \multicolumn{1}{|c|}{ PREGUNTAS } \\
\hline $\begin{array}{l}\text { Percepción } \\
\text { De valor }\end{array}$ & $\begin{array}{l}\text { Percepción del precio de los productos con lo } \\
\text { recibido, ies favorable? } \\
\text { Duración para poder comer, ies aceptable? } \\
\text { ¿El esfuerzo para poder comer vale la pena? }\end{array}$ \\
\hline $\begin{array}{l}\text { Disponibilidad } \\
\text { A volver }\end{array}$ & $\begin{array}{l}\text { Interés en propuesta de regresar a comer } \\
\text { Emoción por volver a comer }\end{array}$ \\
\hline Nivel de exigencia & $\begin{array}{l}\text { Fallas que irritan para no regresar. } \\
\text { Situaciones deleitantes para recomendar el } \\
\text { lugar }\end{array}$ \\
\hline Lealtad & $\begin{array}{l}\text { Salir a comer al mismo restaurante } \\
\text { Ordenar la misma bebida }\end{array}$ \\
\hline Conveniencia & $\begin{array}{l}\text { Comer en el sitio por su cercanía } \\
\text { Menú variado }\end{array}$ \\
\hline
\end{tabular}

Fuente: elaboración propia

Se correlacionaron los 3 indicadores de satisfacción de necesidades (ver Figura 5) junto con el "valor percepción" y la "fidelidad" (asociados a la "Experiencia de comer") para poder determinar cuál peso (se omite por simplificación) se asigna a cada uno de estos indicadores, de manera tal que se obtenga un indicador global de satisfacción, mediante la fórmula siguiente:

\section{I.G.S. $=$ Fisiológico* $0.57+$ Social ${ }^{*} 0.32+$ Intelectual ${ }^{*} 0.11$}

Se puede notar que la satisfacción de necesidades fisiológicas tiene mayor correlación con el "valor percibido" y "fidelidad"; luego se estimó el impacto (\%) de cada indicador que fueron los porcentajes utilizados para ponderar y obtener el indicador global de satisfacción (I.G.S.)
FIGURA 5

\section{VARIABLES CON MAYOR SIGNIFICANCIA ESTADÍSTICA}

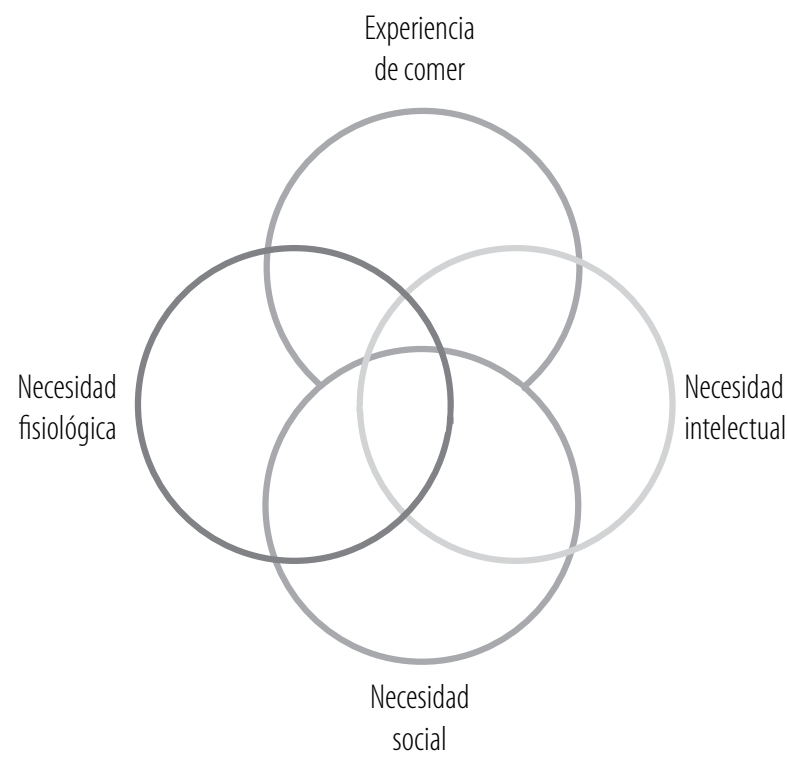

Fuente: elaboración propia

\section{Análisis de resultados de las variables}

Luego de correr la "regresión múltiple" (paso sucesivo) se obtuvo que solamente 3 de las 5 variables del modelo FAMM, tienen mayor correlación sobre la satisfacción de los comensales. En la Tabla 10 se presenta el resumen del modelo obtenido construyendo los indicadores, donde se puede observar el nivel correlacionado con cada variable.

$$
\text { TABLA } 10
$$

\section{RESUMEN DEL MODELO FAMM - MEDIANTE INDICADORES}

\begin{tabular}{|c|c|c|c|c|}
\hline Modelo & $\mathbf{R}$ & R cuadrado & $\begin{array}{c}\text { R cuadrado } \\
\text { corregida }\end{array}$ & $\begin{array}{c}\text { Error típico de } \\
\text { la estimación }\end{array}$ \\
\hline 1 &, $50^{\mathrm{a}}$ &, 25 &, 24 &, 087 \\
\hline 2 &, $57^{\mathrm{b}}$ &, 33 &, 32 &, 083 \\
\hline 3 &, $62^{\mathrm{c}}$ &, 38 &, 36 &, 080 \\
\hline
\end{tabular}

\footnotetext{
a) Variables predictores: (Constante), GERENCIA

b) Variables predictores: (Constante), GERENCIA, ENCUENTRO

c) Variables predictores: (Constante), GERENCIA, ENCUENTRO, OTROS

Fuente: elaboración propia.
} 
Seguidamente, en la Tabla 11, se presenta el modelo obtenido a partir del análisis factorial y que es menos confiable estadísticamente.

TABLA 11

\section{RESUMEN DEL MODELO FAMM - MEDIANTE FACTORIAL}

\begin{tabular}{|c|c|c|c|c|}
\hline Modelo & $\mathbf{R}$ & R cuadrado & $\begin{array}{c}\text { R cuadrado } \\
\text { corregida }\end{array}$ & $\begin{array}{c}\text { Error típico } \\
\text { de la } \\
\text { estimación }\end{array}$ \\
\hline 1 &, $53^{\text {a }}$ &, 28 &, 25 &, 087 \\
\hline
\end{tabular}

a) Variables predictores: (Constante), Comida_Bebida,Servicio_Integral, Contexto,_Control_GERENCIA Fuevie: elaboración propia.

De manera similar, luego se corre el modelo FAMM utilizando las variables obtenidas por el método de los indicadores. Se hicieron 3 regresiones múltiples para cada indicador de necesidades (fisiológica, social e intelectual) a fin de corroborar cuán relacionadas están las variables del FAMM con cada indicador. En la Tabla 12 se resume el efecto de las variables FAMM sobre los indicadores de necesidades. La explicación en cada caso es débil para cada indicador.

Lo anterior implica que el modelo teórico explica una parte de la satisfacción de las necesidades, no obstante, solo lo hacen algunas variables en cada caso. Las variables relacionadas con el "Encuentro", "Control Gerencia" y "Producto" son las que se repiten e interrelacionan con el indicador fisiológico y el intelectual. Vemos que en los 3 indicadores del PAD, el control que ejerce la gerencia tiene su efecto.
Por último, se obtuvo una correlación Pearson entre las variables de los modelos FAMM con el Valor Percibido y Fidelidad; donde resultó que las correlaciones más fuertes se encuentran entre los pares: Valor Percibido_Fidelidad $(0,59)$, Gerencia-Ambiente $(0,54)$, Encuentro-Ambiente $(0,43)$, Producto_Ambiente $(0,45)$, EncuentroAmbiente $(0,43)$, Salón-Ambiente $(0,41)$.

En el $80 \%$ de los casos los aspectos que la gerencia controla pueden hacer sentir bien o muy bien al cliente. Si se considera que de las principales razones para salir, apenas $27 \%$ se relaciona directamente con el placer de comer y ser atendido; mientras que en 31,5\% están asociadas con el hecho "distraerse", ya sea con diversión o socializar. Por otra parte, la encuesta arroja que 32\% de las personas esperan, en esa experiencia de salir a comer, encontrar comodidad durante el evento. Estos hallazgos concuerdan con lo indicado por las teorías relacionadas con el ambiente o atmósfera (Kotler, Ryu, Mehrabian-Russell).

En la muestra se encontró el hecho que 79,7\% de las personas salen al menos una vez al mes a comer a restaurantes, por lo que las percepciones obtenidas sobre el hecho de comer en restaurantes, dan confianza por ser experiencias recientes. Adicionalmente, se contó con la particularidad de que el 75\% de las personas entrevistadas habían visitado por última vez un restaurante hacía menos de un mes; o sea que si la persona encuestada no estaba en ese momento en un restaurante, al menos había estado recien-

TABLA 12

EFECTO DE VARIABLES FAMM SOBRE INDICADORES NECESIDAD (PAD)

\begin{tabular}{|c|c|c|c|c|c|}
\hline Necesidades & FAMM & R & R cuadrado & R cuadrado corregida & Error típ. de la estimación \\
\hline Fisiológica & Encuentro, Gerencia, Producto &, 507 c &, 257 &, 235 &, 10663 \\
\hline Social & Otros, Ctrl. Gerencia &, $546^{\text {b }}$ &, 298 &, 285 &, 10589 \\
\hline Intelectual & Encuentro, Gerencia, Producto &, 504 c &, 254 &, 232 &, 18977 \\
\hline
\end{tabular}

Fuente: elaboración propia. 
temente en uno y se espera que al responder, la experiencia fuera aún bastante clara.

Entre los hábitos de visita a restaurantes del estudio, se encontró que más de la mitad de las personas $(56,2 \%)$ en la muestra visitan este tipo de restaurantes con su pareja. En caso de salir en grupo, es típico que esté integrado por familiares (39\%), personas similares con las que se identifica $(26,7 \%)$ o viejos amigos (excompañeros-clases) $24,8 \%$.

Además, la mayoría prefiere como actividad recreativa salir a comer a restaurantes, tanto entre semana $(45,7 \%)$ como en fin de semana $(42,9 \%)$. La segunda actividad preferida entre semana es ver películas $(28,6 \%)$ y en fin de semana el visitar comercios $(17,1 \%)$.

En las preferencias respecto a la atmósfera, el $46,7 \%$ prefiere algo tranquilo, un $20,0 \%$ gusta de lo armonioso, a un 21,9\% le es indiferente y solo al 1,0\% le gusta lo bullicioso. Además que cuando salen a comer, en un $44,8 \%$ a los sujetos del grupo muestreado también les gusta disfrutar el ambiente, mientras que otros durante la comida les gusta ver el paisaje $(17,1 \%)$, así como al 10,5\% conversar con personal del restaurante.

Cuando se preguntó el tipo de festejo que les hace ir a un restaurante, el mayor fue por evento familiar $(44,8 \%)$ seguido de cumpleaños $(23,8 \%)$ y luego aniversario de pareja $(21,9 \%)$. Basado en los datos, se considera que los elementos tecnológicos infaltables en un restaurante son: música (40,0\%), Wi-fi $(21,0 \%)$ y T.V. (12,4\%). Los eventos televisivos que más prefieren ser vistos en un restaurante, según resultados son de tipo deportivo; temporadas de carácter internacional (liga-baseball, la champions league, euro-copa) con un 24,8\% y la selección de fútbol (22,9\%)

Las principales fallas por las cuáles no regresarían a un restaurante, según la opinión de los sujetos fue: la calidad del servicio $(61,0 \%)$ y los productos (27,0\%); en segundo plano la eficiencia
$(6,7 \%)$ y, por último, algún problema con el ambiente $(3,8 \%)$ o confort en instalaciones $(2,9 \%)$.

Respecto a los atributos del producto, un 67,6\% considera el sabor como el más importante, seguido por el tamaño de la porción con 9,5\% y presentación 8,6\%; quedando en un tercer plano la temperatura $(3,8 \%)$ y textura $(2,9 \%)$. Los comensales encuestados prefieren pedir de comer: "plato principal + postre" $(30,5 \%)$, "entrada + plato principal + postre" (28,6\%), "solo plato principal" $(26,7 \%)$ y"entrada + plato principal" (14,3\%).

En la opinión de los encuestados, consideraron como aspectos claves del servicio: el servicio oportuno de los productos (45,7\%), la atención a sus solicitudes (22,9\%), la interrelación del personal (14,3\%) y la toma de la orden (8,6\%); queda en un segundo plano la facturación correcta y oportuna $(2,9 \%)$ y la forma de pago (1,9\%). A pesar que el tema de facturación muestra baja importancia, un 37,1\% indica sentirse irritado en caso de retraso, un 19,0\% decepcionado, 16,9\% enojados, 13,3\% escépticos y $8,6 \%$ dijeron sentirse frustrados.

Respecto a lo clave de la interrelación con el personal, se observó que los tres con mayor porcentaje son: un 30,5\% es la amabilidad y empatía, $26,7 \%$ el conocimiento de productos y $20,0 \%$ la toma de orden y servicio de productos oportunamente.

Los tipos de bebidas para acompañar la comida encontrados fue que un $47,6 \%$ prefiere refrescos naturales, $21,0 \%$ alguna bebida con licor, un $13,3 \%$ refrescos gaseosos y $8,6 \%$ agua.

\section{CONCLUSIONES}

Hasta el momento de realización de la investigación, no se encontró estudio similar para el sector gastronómico aquí analizado, donde las ciencias administrativas utilicen la rigurosidad científica de base para la toma de decisiones. Dados los antecedentes encontrados es preciso realizar una investigación a mayor profundidad 
para llenar el vacío encontrado que resulte en información útil para ese sector empresarial.

Las variables bajo control de la gerencia tienen el mayor impacto sobre la percepción de la experiencia de los clientes que visitan el tipo de restaurante analizado. Otras variables importantes se relacionan con el mero placer de comer y ser atendido; por tanto, los empresarios deben poder contar con la suficiente información confiable acerca de las motivaciones de sus clientes para que basado en esto, logren realizar una exhaustiva planificación que les conduzca a una excelente ejecución en el momento que se brinda el servicio.

Es muy viable, por medio de la administración de las variables que controla la gerencia, crear esas experiencias satisfactorias que el visitante espera recibir en el lugar. Tener certeza de cuáles son esas principales variables, ayudará también al empresario de ese nicho gastronómico a conocer y priorizar las inversiones necesarias para satisfacer las expectativas de sus comensales. A partir de esa claridad, el dueño podrá tomar sus decisiones estratégicas sobre el negocio, ya sean estas invertir capital, retirarse u otra acción según los intereses de los socios.

Si se quisiera tomar algunas decisiones sobre este trabajo, los empresarios deben invertir en elementos que sirvan para entretener a los clientes mientras comen, así como garantizar unas instalaciones cómodas para que disfruten mejor su estadía a lo largo del evento y buenas atenciones a sus solicitudes durante el proceso de servicio. Está claro que los atributos del producto deben mantenerse al nivel de calidad requerido, ya que un $67,7 \%$ de comensales, tiene sus expectativas en el sabor del producto, seguido por el tamaño de la porción y presentación respectivamente en un $9,5 \%$ y $8,6 \%$.

Un estudio de este tipo brinda información importante para decidir la variedad a ofrecer en el menú, prestando atención a los hábitos de consumo. Una estrategia valiosa puede ser mante- ner un buen balance de opciones de postres y entradas. Puntualizando como ejemplo, los resultados de la muestra, la preferencia al ordenar es de un 30,5\% "plato principal + postre", un 28,6\% "entrada + plato principal + postre"; por lo que el empresario que conozca esta información estará buscando cómo ofrecer en su menú más alternativas para satisfacer las preferencias en postres $(59,1 \%)$ con mayor atención que de las entradas (42,9\%).

Los aspectos de comunicación (medios publicitarios, frecuencia de mensajes) para llegar al perfil de la muestra deben ser parte de las estrategias del sector empresarial, buscando cómo lograr llegarle a esos clientes meta, pues para el 79,7\% salen al menos una vez por mes y además es la actividad recreativa preferida, tanto entre semana $(45,7 \%)$ como fin semana. De esta manera el comprador lo tendrá presente al momento de elegir dónde comer y tener opciones para facilitar áreas para compartir tanto en pareja como en grupos. Al realizar esa comunicación es primordial transmitir al potencial consumidor, las imágenes del ambiente que despierte interés en visitar el local, así como brindar información suficiente para influir en su decisión de probar los productos y servicios ofrecidos dejando claro en el mensaje la atmósfera en que serán recibidos.

También la información brindada por el instrumento es de utilidad para un inversionista que desee incursionar en dicho segmento. Véase por ejemplo que en los aspectos tecnológicos que se disponen en el tipo de restaurante analizado, los comensales opinan, respecto a "elementos tecnológicos infaltables". Ellos esperan encontrar en un 40,0\% música, en un $21,0 \%$ de los casos se espera contar con Wi-fi y 12,4\% T.V. Además, al cruzar esta última información con el hecho que entre los "eventos televisivos que más prefieren ver en un restaurante", están los deportivos 47,7\% razón por la cual una decisión apoyada en esto implica mantener en los televisores algún canal exclusivo que transmita deportes. 
Muy delicado es el tema de lograr satisfacer las expectativas comunicadas en una campaña publicitaria o en el boca a boca; donde la gerencia ha de poner muchísima atención en la capacitación y entrenamiento de su personal del salón para hacer realidad la percepción que lleva el comensal desde que tomó su decisión de comer en el restaurante de su elección y visita. Los resultados indican las principales fallas por las cuáles no regresaría el cliente a un restaurante, siendo estas en un 61,0\% las de calidad del servicio, seguida por los productos en el 27,0\% de los casos. Véase como al cruzar esta información con el hecho que el servicio oportuno del producto en un $45,7 \%$ es considerado un aspecto clave del servicio, lo cual obliga al gerente a tomar acciones concretas en su sistema para hacer realidad la satisfacción de tal aspecto si se quiere lograr que el comensal quiera repetir la experiencia de su visita.

Igualmente, en un estudio como el presente, se obtiene información suficiente para planear un programa de capacitación del personal de las distintas áreas (salón, bar, caja, parqueo) que está en contacto directo con los clientes. Le idea es que dicho personal esté preparado para una interrelación amable y empática con el cliente, que el personal del salón tenga dominio pleno de los productos; así como contar con una organización y operación que esté en capacidad de poder ofrecer productos servidos oportunamente.

Es posible conducir otros estudios a partir de los hallazgos encontrados para ampliar la profundidad de información en aquellos aspectos más relevantes. La base de datos en SPSS permite realizar otros análisis, cruzando datos para los distintos grupos demográficos y obtener información interesante, como por ejemplo, el comportamiento de cierto rango de edad o sexo ante alguna de las variables analizadas con mayor significancia.

\section{RECOMENDACIONES}

Las estrategias de mercadeo para un restaurante del tipo analizado, debería contener un fuerte componente que atraiga al consumidor a compartir, es decir destacar los criterios sociales que puedan generarse en el lugar e invitar a una agradable experiencia.

Con respecto al personal, es recomendable que la gerencia de un restaurante del tipo "servicio completo", realice una selección de su personal de salón con cualidades de servicio, habilidades actitudinales para estar motivado a pesar del estrés que suele manejarse en horas pico. Debe darse importancia a los planes de entrenamiento del personal de cocina, según las competencias necesarias para hacer realidad la oferta de productos con alta calidad y la suficiente variedad de platillos del menú.

La gerencia ha de tomar las medidas necesarias para que el sistema sea eficiente y listo para atender oportunamente las solicitudes de clientes; $85 \%$ de los encuestados lo confirman.

Un 31\% de la muestra desea distraerse al salir, la evidencia refleja que la manera en que esperan hacerlo es divirtiéndose o socializando; se recomienda a los empresarios tomar esto como una oportunidad para la administración de transformar esta información en fortaleza para mantener a los clientes. De manera similar, se sugiere que el diseño de las instalaciones (exteriores e interiores) puedan facilitarle a la clientela diversas oportunidades para sentirse confortables, esto según los resultados que muestran que un 32\% de las personas están esperando que su experiencia de salir les depare diversas comodidades.

Para realizar estudios con estadística más contundente, se ha de incrementar el número de la muestra y así incrementar una mayor confiabilidad estadística. 
Las necesidades fisiológicas están muy correlacionadas con "valor percibido" y "fidelidad", al cual le sigue el "social". Estas son pautas por considerar por la gerencia cuando comunica en sus medios de publicidad, el producto que será consumido; si se tiene muy presente que el comensal le será muy fiel en la medida que logre satisfacer en alto grado su necesidad de alimentarse y si logran hacerlo en un ambiente socialmente agradable, la experiencia querrá ser repetida por el visitante.

Elementos controlados por la gerencia son los que mantienen la "fidelidad" y un "ambiente" adecuado. Por ende, en buena medida los criterios de ejecución (proceso de toma de los pedidos, tiempos de espera de los alimentos y proceso de cancelación) deben ser supervisados para garantizar sean realizados con excelencia; de forma similar, la atención y el mantenimiento del entorno (música, apoyos tecnológicos, ventilación e iluminación, por ejemplo) que le permita al comensal degustar sus alimentos de manera confortable.

Se recomienda realizar otro estudio similar en otra zona del país para poder contrastar los resultados con la presente investigación y evaluar si los comportamientos son análogos o hay grandes diferencias que lleven a plantear estrategias diferentes en los mismos tipos de restaurantes localizados en zonas distintas.

\section{GLOSARIO}

Restaurante tipo servicio-completo: es aquel en el cual los huéspedes son recibidos y sentados por un host (o anfitriona) y los pedidos son tomados y entregados por personal de servicio (meseros); el pago se hace después que la comida ha terminado y una propina se da normalmente a los meseros por el servicio recibido.

Salón: abarca los factores presentes en el salón que impactan de alguna manera en la percepción del comensal como iluminación, sonido colores, diseño, música, decoración, T.V.Mifi y materiales, entre otros.

Encuentro: son las interacciones del cliente a medida que encuentra a otras personas; son muy importantes las relaciones cordiales, la buena comunicación, el acogimiento y la empatía con sus interlocutores; algunos detalles son: guarda-parqueo, anfitriones, otros comensales, meseros en el bar, reglas de etiqueta y cajera(o), por ejemplo.

Producto: son los distintos atributos de cada comida y bebida, que podrían comunicarse mediante descripción o fotografías; los principales son: presentación en el plato, tamaño de la porción, apariencia, temperatura, sabor, decoración, envase/cristalería, estado de la mezcla y balance del licor, por citar algunos.

Sistema de control gerencial: se refiere a cómo está organizado el restaurante en sus distintos elementos administrativos y, además, su forma de operar; hay grandes diferencias si es un buffette, a la carta o comida rápida por ejemplo; todas estas variables se notan en el sistema administración, el tiempo de servicio de los productos, el liderazgo, el control de rotación, los procedimientos, las normas y el código conducta, la logística en cocina, la logística en salón, la forma de pago, el tomado de orden, el contacto e-mail, la facturación (OTIF), el servicio simultáneo de productos y el menú (información y diseño).

Atmósfera/ambiente: esta variable es a su vez "independiente" y "dependiente", e interviene en la experiencia; es bastante abstracta e intangible. Según Watz (2008), la dinámica depende de las otras variables estudiadas (comida, música, olor en el aire y demás). O sea, el ambiente es a la vez influenciado por diversas variables, pero genera una percepción determinada que influye en la experiencia del cliente.

Necesidades fisiológicas: basadas en satisfacer las necesidades de comer y beber para el caso de restaurantes. 
Necesidades sociales: parcialmente basadas en satisfacer pertenencia a grupos, autoestima, jerarquía dentro de esos grupos y que pueden ser satisfechas mediante membresía, símbolos de estatus, títulos y consumo conspicuo.

Necesidades intelectuales: están basadas en toda fuente de interés, entretenimiento y lo excitante; peligro y novedad de determinado grado puede ser ingredientes importantes. Dichas necesidades pueden ser satisfechas por un trabajo gratificante, música, literatura, ver deportes, juegosapuesta, arte y otros.

\section{Variables dependientes (criterios de las variables)}

Definición de variables que se van a estudiar: la variable dependiente "Consumo"

- Intensidad: que nos va a decir qué tanto el cliente permanece en el sitio.

- Frecuencia: se refiere a las veces que repite la visita al restaurante.

- Intencionalidad: es la intención u objetivo que tiene el cliente para visitar el lugar.

- Variedad: los distintos productos que consume

- Ocasión: las ocasiones en que el cliente se presenta a consumir (ej. cumpleaños, día de la madre y otros)

\section{Explicación de las variables modelo FAMM}

Aspectos del Salón

- $\quad$ Iluminación $\rightarrow$ Natural, artificial, tonos de las luces, intensidad, directa e indirecta.

- $\quad$ Sonido $\rightarrow$ Tipos (cristalería, el de otros comensales, equipos presentes, externo, sillas que se arrastran) el nivel percibido (muy bajo, bajo, medio, alto, muy alto)
- Colores $\rightarrow$ Son los que hay en diversos elementos del salón (color paredes, el de los manteles, techo, muebles)

- Diseño $\rightarrow$ En dependencia del tipo -Abierto, cerrado, ventanales (si, no con visibilidad hacia fuera, efecto en la circulación del aire y olores)-, causará distinto efecto sobre los comensales y sus productos.

- $\quad$ Música $\rightarrow$ Se refiere a la amenización musical que se ofrece a sus clientes, en vivo, reproducida, el género, variedad, cíclica o repetitiva, origen (nacional, internacional).

- Decoración $\rightarrow$ Los detalles en diversos sitios del salón (adornos, cuadros-pintura, esculturas, ocasionales o de temporada, rotulaciones, espejos y otros)

- $\quad$ T.V./Wifi $\rightarrow$ Disposición de t.v., tipo de programa o canal ofrecido, videos musicales, documentales transmitidos, información del mismo sitio. Se dispone de zona Wifi, es para todos, solo clientes frecuentes, hay clave o es abierto.

- Materiales $\rightarrow$ Elementos de construcción del interior, sus divisiones, gradas, puertas (madera, metal, vidrio)

\section{Aspectos del Encuentro}

- Guarda-parqueo $\rightarrow$ ¿Hay cuidador de vehículos? ¿Saluda cordialmente?

- Hostess $\rightarrow$ Persona quién recibe a los clientes y ubica en mesas, nivel cordialidad.

- $\quad$ Otros comensales $\rightarrow$

- Meseros $\rightarrow$ Cortesía, nivel de empatía, nivel de comunicación, presentación.

- $\quad$ En el bar $\rightarrow$ similar a meseros, pero quizás más conversación, ya que suelen atender a clientes que van solos.

- Reglas de etiqueta $\rightarrow$ Personal en general sigue normas de etiqueta,

- $\quad$ Cajera(o) $\rightarrow$ Brinda servicio para facturación. 
Aspectos del Producto

- Presentación en el plato $\rightarrow$ según como viene distribuida la comida en el plato puede causar impresión positiva o negativa para cliente, la forma del plato y los espacios.

- Tamaño - porción $\rightarrow$ esto se relaciona con las expectativas del comensal y se aplica tanto a bebidas como a la comida.

- Apariencia $\rightarrow$ suele estar influenciada por la iluminación.

- Temperatura $\rightarrow$ es clave y estar fuera de los rangos puede causar mucha disconformidad al cliente, aplicable en las bebidas y comidas.

- Sabor $\rightarrow$ elemento básico para el deleite o no del cliente, la memoria guarda un recuerdo asociado a un platillo y bebida, el cual se refuerza con cada visita.

- Decoración $\rightarrow$ cómo se adorna cada plato o bebida, entre más conozca el cliente por experiencias previas, se formará una expectativa sobre este elemento.

- Envase/cristalería $\rightarrow$ la forma del envase, hay estándares asociados con ciertas bebidas, limpieza y nitidez asociada con higiene.

- Estado -mezcla $\rightarrow$ en las bebidas se refiere a si es "frozen", se espera así y no en otro estado.

- Balance licor $\rightarrow$ al igual que con el sabor, la persona tiene una expectativa acorde a su estándar, esto varía según el sitio y una vez degustado ahí, se guarda la memoria de la cantidad sentida de licor.

\section{Aspectos del Sistema de Control Gerencial}

- $\quad$ Sistema administración $\rightarrow$ se rige por metas, hay establecidos incentivos como el empleado del mes y otros mecanismos para administrar el negocio y motivar al personal.

- $\quad$ Tiempo-servicio de productos $\rightarrow$ duración prevista para brindar el servicio de los productos al comensal desde que ordena hasta que se los sirven.
- $\quad$ Liderazgo $\rightarrow$ el tipo de liderazgo que afecta la motivación, productividad y capacidad del personal en general.

- Control rotación $\rightarrow$ para garantizar insumos frescos en la preparación de los alimentos.

- $\quad$ Procedimientos $\rightarrow$ cumplimiento con estándares de HACCP, así como buenas prácticas en la manipulación de alimentos y otros para tratar con quejas o molestias de clientes ante eventuales situaciones.

- Normas/código conducta $\rightarrow$ el esquema que rige o pone en práctica el personal en sus labores.

- Logística cocina $\rightarrow$ forma de operar en la cocina y la manera de procesar las órdenes de clientes para procurar brindar una atención conforme el tipo de restaurante. Esto tiene su impacto en los tiempos de respuesta a los pedidos que salen.

- Logística salón $\rightarrow$ la distribución de mesas, asignación por mesero, los apoyos para brindar el servicio (ej. hay 1 servilletero por mesa o una batería que se asigna conforme llegan clientes, las mesas se mantienen montadas o se preparan hasta que llega el comensal) y otros elementos.

- $\quad$ Forma de pago $\rightarrow$ efectivo, crédito (cualquier tarjeta, restringidas), descuento por cliente frecuente.

- Tomado de orden $\rightarrow$ tecnología utilizada,

- $\quad$ Contacto e-mail $\rightarrow$ atención post-venta o promociones, comunicaciones, publicidad.

- $\quad$ Facturación (OTIF) $\rightarrow$ tiempo de respuesta, nivel de errores, atención para resolver problemas o solicitudes de clientes.

- Servicio simultáneo de productos $\rightarrow$ se sirven conforme solicitan, se entregan los productos a la vez o como van saliendo de la cocina.

- Menú (Infor./Diseño) $\rightarrow$ tipo de menú (ilustrado, solo texto, información nutricional, desechable, materiales) 


\section{REFERENCIAS}

Acharya, R., Patterson, P., Schmitz, T., Foerster, S., Hill, E., Jones, A. y Bohm, E. (2002). Restaurant Diners' Attitudes and Responses to a Healthy Dining Campaign.California: American Agriculture Economics Association 2002 Annual Meeting, Selected Paper.

Anderson, T. y Mossberg, L. (2004). The dining experience: do restaurants satisfy customer needs. Journal of Food Service, (4), 171-177

Alonso, A. y O’Neill, M. (2010). To what extent does restaurant kitchen design influence consumers' eating out experience? An exploratory study. Journal of Retail y Leisure Property

Banco Central de Costa Rica. (2011). Evolución del PIB real y la balanza de pagos, I trimestre 2011. Recuperado de http://indicadoreseconomicos. bccr.fi.cr/indicadoreseconomicos/Documentos/Sector\%20Producci\%C3\%B3n\%20y\%20Empleo/Evoluci\%C3\%B3n\%20PIB\%20trimestral/ Evoluci\%C3\%B3n\%20PIB\%20Real\%20y\%20Balanza\%20de\%20Pagos\%20l\%20Trimestre\%202011.pdf

Edwards, J. y Gustafsson, I. (2008) The Five Aspects Meal Model. Journal of Food Service, 19(1), 4-12.

Han, H., Back, K. y Barrett, B. (2008). A Consumption Emotion Measurement Development: A Full-Service Restaurant Setting. The Service Industries Journal, 30(2), 299-320.
Howlett, E., Burton, S., Bates, K. y Huggins, K. (2010). Coming to a Restaurant Near You? Potential Consumer Responses to Nutrition Information Disclosure on Menus. Journal of Consumer Research, Inc.

Instituto Nacional de Estadística y Censos (INEC). (2007). Panorama Demográfico. Boletín Anual; Vol. 1, Año 1.

Instituto Nacional de Estadística y Censos (INEC). (2006) Encuesta Nacional de Ingresos y Gastos de los Hogares 2004. San José.

Ryu, K. (2005). Dinescape, Emotions And Behavioral Intentions In Upscale Restaurants. Manhattan, Kansas. ProQuest Information and Learning Company

Schembri, S; Sandberg, J; (2011) The experiential meaning of service quality; Journal Marketing Theory.

Watz, B. (2008).The entirety of the meal: a designer's perspective. Journal of Food Service.

Worsfold, D. (2006). Eating out: Consumer perceptions of food safety. International Journal of Environmental Health Research.

Recibido: 9 de mayo de 2013 Aceptado: 15 de octubre de 2013 